\title{
7. Über die Guanidinderivate, welche auf den Blutzucker senkend wirken.
}

Von Taizo Kumagar, Sin-iti KawaI, und Yoshio ShIKInami. Aus dem chemischen Institut und der medizinischen Klinik der Tohoku Universität zu Sendai.

(Rec. Dec. 12, 1927. Comm. by R. Majima M.I.A., Dec. 12, 1927.)

1918 bemerkte C.K. Watanabe, ${ }^{1)}$ dass das Guanidin eine auffallende Hypoglykämie erzeugt. Im Anschluss an diese Untersuchung haben Frank, Nothmann und Wagner ${ }^{2}$ ein wirksames Derivat des Guanidins dargestellt, welches sie Synthalin nannten. Das Synthalin soll in der Menge von $3 \mathrm{mg}$ auf den Blutzucker des Kaninchens herabsetzend wirken.

Wir haben ähnliche Versuche mit mehreren Guanidinderivaten in Angriff genommen, um den Mechanismus der blutzuckersenkenden Guanidinwirkung näher aufklären zu können. Trotzdem unsere Untersuchung noch nicht abgeschlossen, vor allem die Darstellung des Oktaund Dekamethylendiguanidins aus Sebacinsäure noch im Gange ist, wollen wir doch unsere bisherige Untersuchung veröffentlichen, weil die Konstitution des Synthalins neuerdings als Dekamethylendiguanidin von Frank ${ }^{3)}$ angegeben ist. Allerdings hat das von uns dargestellte Hexamethylendiguanidin ebenso starke Wirkung wie das erwähnte Synthalin.

Die Präparate von Nr. 1-10 sind von Prof. R. Majima ${ }^{4)}$ dargestellt und schon veröffentlicht. Nr. 11-12 sind von K. Suminokura, Nr. 13 von $\mathrm{K}$. Suminokura und S. Kawai, und Nr. 14-15 von S. Kawai synthetisiert worden, aber noch nicht mitgeteilt. Die letzteren sollen in kurzem in "Scientific Papers of the Physical and Chemical Research Institute, Tokyo" publiziert werden.

Die anderen Guanidinderivate von Nr. 16-22 sind von uns durch die Kondensation von S-Methyl-pseudothioharnstoff und entsprechenden Aminen dargestellt. Zur Darstellung des Agmatins (Nr. 17) liess man

1) C.K. Watanabe: Journ. of biol. chemist., Bd. 33, S. 253, 1918.

2) Frank, Nothmann und Wagner: Klin. Wochenschr., Jg. 5, Nr. 45, S. 2100. 1926.

3) Frank: Deut. med. Wochenschr., Jg. 53, Nr. 43, S. 1845, 1927.

4) R. Majima : Ber, Bd. 41, S. 176, 1908. 
1 Mol jodwasserstoffsauren S-Methyl-pseudothioharnstoff auf $1 \mathrm{Mol}$ Putrescin einwirken. Man beseitigte von dem festen Reaktionsprodukte unverändert gebliebenes Putrescin mit Äther. Als man die so entstandenen jodwassertoffsauren Salze nach bekannter Weise in schwefelsaure Salze umgewandert hatte, kristallisierte zuerst das unreine Tetramethylendiguanidinsulfat (Nr. 18), darauf das reine Agmatinsulfat ${ }^{1)}$ (Schm. p. 228 ${ }^{\circ}$. Das Tetramethylendiguanidinsulfat wurde als solches zum physiologischen Versuch verwendet, weil die Reindarstellung desselben wegen geringer Menge schwer ausführbar war.

Auf dieselbe Weise versuchten wir aus $1 \mathrm{Mol}$ Cadaverin und $1 \mathrm{Mol}$ S-Methyl-pseudothioharnstoff, 5-Amino-pentamethylenguanidin, eine höhere Homologe von Agmatin, darzustellen. Trotz mehrere zehn Male wiederholter Umkristallisation ist die Reindarstellung des Präparates nicht gelungen, wie es schon Otto Ripke ${ }^{2}$ erfahren hat. Dabei konnten wir als Nebenprodukt Pentamethylendiguanidinsulfat (Nr. 19) (Z.p. $330^{\circ}$. N. gef. 29.33 , ber. 29.57) rein gewinnen.

Zuerst wurde Hexamethylendiamin nach v. Braun und Müller) aus Piperidin dargestellt, dann 1 Mol Hexamethylendiamin und 2 Mole jodwasserstoffsaurer S-Methyl-pseudothioharnstoff zusammengebracht, das entstandene jodwasserstoffsaure Hexamethylendiguanidin erst mit Silberkarbonat, dann mit $\mathrm{SH}_{2}$ behandelt. Das Filtrat von Schwefelsilber wurde mit Salzsäure angesäuert. Die unreinen höher schmelzenden Substanzen wurden wiederholt abfiltriert; aus der Mutterlauge ist das fast reine Hexamethylendiguanidinhydrochlorid (Nr. 22) gewonnen. Umkristallsiert aus Alkohol $+\ddot{A}$ ther, schmilzt bei $175-176.5^{\circ}$.

Untersucht wurde hauptsächlich die den Blutzucker senkende Wirkung dieser Guanidinderivate beim Kaninchen, indem wir sie diesem subcutan oder peroral einverleibten.

12 Guanidinpräparate - C-Diäthyl - malonyl - guanidinhydrochlorid (Nr. 2), Acetylaceton-guanidinhydrochlorid (Nr. 3), $\beta$-Dimethyl-imidouracilhydrochlorid (Nr. 4), $\alpha$-Di-methyl-imido-uracilhydrochlorid (Nr. 5.), Acetylaceton-methylguanidinhydrochlorid (Nr. 6), $\beta$-Allylmethylimido-uracilhydrochlorid ( $\mathrm{Nr} .7$ ), C-Di-äthyl-malonyl-methyl-guanidinhydrochlorid (Nr. 8), $\alpha$-Allyl-methyl-imido-uracilhydrochlorid (Nr. 10), 2-3-Trimethylen-imino-4-oxo-6-methyl-tetrahydropyrimidinhydrochlorid (Nr. 11), Trimethylenguanidinsulfat (Nr. 12), 2-3-̈̈thylen.imino-6-

1) Vergl. nach Kossel (H. Bd. 68, S. 170, 1910. Schm. p. 229 .) und nach Kiesel (H. Bd. 118, S. 279, 1922. Schm. p. 226. .)

2) O. Ripke: H. Bd. 72, S. 484, 1911.

3) Braun und Müller: Ber. Bd. 38, S. 2204, 1905. 
methyl-4-oxo-tetrahydropyrimidinhydrochlorid (Nr. 13) und 2-Oxyäthylimino-6-methyl-4-oxo-tetrahydropyrimidinhydrochlorid ( $\mathrm{Nr}$. 15) -, in denen beide $\mathrm{NH}_{2}$-Gruppen des Guanidinmoleküls besetzt sind, haben bei der Einverleibung von $0,1-0,2 \mathrm{~g}$ pro $\mathrm{kg}$ keine merkbare Wirkung erwiesen.

Was die Wirkung von Guanidinderivaten - Methylguanidinsulfat (Nr. 9), $\beta$-oxy-äthylguanidinbromhydrat (Nr. 14), $\beta$-( $\beta^{\prime}$-Indolyl)-äthylguanidinhydrochlorid (Nr. 16), Allylguanidinsulfat (Nr. 1), Propylguanidinsulfat (Nr. 20) und Agmatinsulfat (Nr. 17) - , bei denen nur eine $\mathrm{NH}_{2}$-Gruppe frei vorhanden, anbetrifft, nimmt die blutzuckerherabsetzende Wirkung mit der Verlängerung der $\mathrm{CH}_{2}$-Kette allmählich $\mathrm{zu}$, während die Giftigkeit nach und nach abnimmt; die Verstärkung der blutzuckersenkenden ist aber dabei nicht so gross; bei Agmatin, welches $4 \mathrm{CH}_{2}$-Ketten hat, ist seine Wirkung nur doppelt so stark wie bei den Präparaten mit $2 \mathrm{CH}_{2}$-Ketten, und gleiches Ergebnis zeigen die Derivate mit $3 \mathrm{CH}_{2}$-Ketten.

In der folgenden Tabelle seien die Resultate mit den Polymethylendiguanidinen, bei denen je ein freies Guanidinmolekül an den beiden Enden der $\mathrm{CH}_{2}$-Kette vorhanden ist, angegeben.

Wie man aus der Tabelle ersieht, ist die Wirkung des Äthylendiguanidins ungefähr doppelt so stark wie bei $\beta$-oxy-äthylguanidin. Tetramethylendiguanidin hat ganz wenig stärkere Wirkung wie bei Äthylendiguanidin, was wahrscheinlich dem nicht ganz reinen Präparat zuzuschreiben wäre. Beim Pentamethylendiguanidin ist die Wirkung auffallend verstärkt, und fast fünffach so gross wie die des vorigen.

Wenn man die $\mathrm{CH}_{2}$-Kette bis auf 6 verlängert, so ist die Wirkung fast zehnfach stärker als die des Präparates mit $5 \mathrm{CH}_{2}$-Ketten.

Zum Vergleich haben wir uns Synthalin aus Deutschland kommen lassen ; das Resultat desselben ist auch in derselben Tabelle angeführt, ebenso die Ergebnisse der peroralen Applikation von Penta- , Hexamethylendiguanidin und Synthalin. Bei der peroralen Applikation haben wir im Vergleich mit der subkutanen keinen Unterschied finden können. Und das Resultat zeigt, dass das käufliche Synthalin, so wohl bei subkutaner wie auch bei peroraler Applikation, gar nicht stärker als Hexamethylendiguanidin ist.

Zum Schluss sprechen wir Herrn Prof. Dr. R. Majima für seine freundliche Hilfe unseren verbindlichsten Dank aus. 


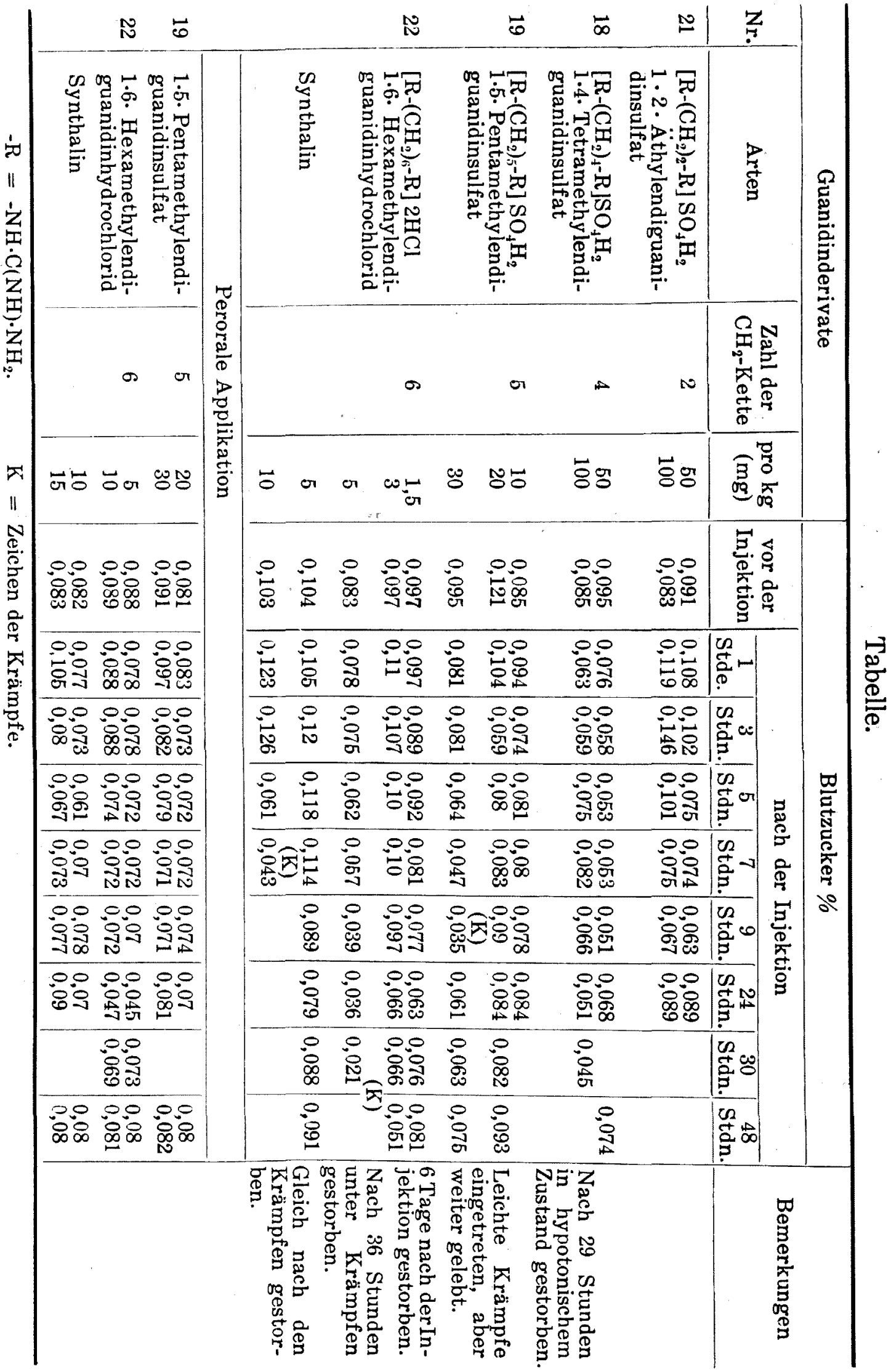

